\title{
CHARACTERIZING LINEAR MAPPINGS THROUGH ZERO PRODUCTS OR ZERO JORDAN PRODUCTS
}

\author{
GUANGYU AN ${ }^{1}$, JUN HE ${ }^{2}$ AND JIANKUI LI* ${ }^{3 *}$
}

\begin{abstract}
Let $\mathcal{A}$ be a $*$-algebra and $\mathcal{M}$ be a $*$ - $\mathcal{A}$-bimodule, we study the local properties of $*$-derivations and $*$-Jordan derivations from $\mathcal{A}$ into $\mathcal{M}$ under the following orthogonality conditions on elements in $\mathcal{A}: a b^{*}=0, a b^{*}+b^{*} a=0$ and $a b^{*}=b^{*} a=0$. We characterize the mappings on zero product determined algebras and zero Jordan product determined algebras. Moreover, we give some applications on $C^{*}$-algebras, group algebra, matrix algebras, algebras of locally measurable operators and von Neumann algebras.
\end{abstract}

\section{INTRODUCTION}

Throughout this paper, let $\mathcal{A}$ be an associative algebra over the complex field $\mathbb{C}$ and $\mathcal{M}$ be an $\mathcal{A}$-bimodule. For each $a, b$ in $\mathcal{A}$, we define the Jordan product by $a \circ b=a b+b a$. A linear mapping $\delta$ from $\mathcal{A}$ into $\mathcal{M}$ is called a derivation if $\delta(a b)=a \delta(b)+\delta(a) b$ for each $a, b$ in $\mathcal{A}$; and $\delta$ is called a Jordan derivation if $\delta(a \circ b)=a \circ \delta(b)+\delta(a) \circ b$ for each $a, b$ in $\mathcal{A}$. It follows from the results in $[9,20,21]$ that every Jordan derivation from a $C^{*}$-algebra into its Banach bimodule is a derivation.

By an involution on an algebra $\mathcal{A}$, we mean a mapping $*$ from $\mathcal{A}$ into itself, such that

$$
(\lambda a+\mu b)^{*}=\bar{\lambda} a^{*}+\bar{\mu} b^{*},(a b)^{*}=b^{*} a^{*} \text { and }\left(a^{*}\right)^{*}=a,
$$

whenever $a, b$ in $\mathcal{A}, \lambda, \mu$ in $\mathbb{C}$ and $\bar{\lambda}, \bar{\mu}$ denote the conjugate complex numbers. An algebra $\mathcal{A}$ equipped with an involution is called a $*$-algebra. Moreover, let $\mathcal{A}$ be a $*$-algebra, an $\mathcal{A}$-bimodule $\mathcal{M}$ is called a $*-\mathcal{A}$-bimodule if $\mathcal{M}$ equipped with a $*$-mapping from $\mathcal{M}$ into itself, such that

$$
(\lambda m+\mu n)^{*}=\bar{\lambda} m^{*}+\bar{\mu} n^{*},(a m)^{*}=m^{*} a^{*},(m a)^{*}=a^{*} m^{*} \text { and }\left(m^{*}\right)^{*}=m,
$$

whenever $a$ in $\mathcal{A}, m, n$ in $\mathcal{M}$ and $\lambda, \mu$ in $\mathbb{C}$. An element $a$ in $\mathcal{A}$ is called self-adjoint if $a^{*}=a$; an element $p$ in $\mathcal{A}$ is called an idempotent if $p^{2}=p$; and $p$ is called a projection if $p$ is both a self-adjoint element and an idempotent.

In [24], A. Kishimoto studies the $*$-derivations on a $C^{*}$-algebra. Let $\mathcal{A}$ be a *-algebra and $\mathcal{M}$ be a $*-\mathcal{A}$-bimodule. A derivation $\delta$ from $\mathcal{A}$ into $\mathcal{M}$ is called a *-derivation if $\delta\left(a^{*}\right)=\delta(a)^{*}$ for every $a$ in $\mathcal{A}$. Obviously, every derivation $\delta$ is a linear combination of two $*$-derivations. In fact, we can define a linear mapping $\hat{\delta}$ from $\mathcal{A}$ into $\mathcal{M}$ by $\hat{\delta}(a)=\delta\left(a^{*}\right)^{*}$ for every $a$ in $\mathcal{A}$, therefore $\delta=\delta_{1}+i \delta_{2}$, where

2010 Mathematics Subject Classification. 15A86, 47A07, 47B47, 47B49.

Key words and phrases. *-(Jordan) derivation, *-(Jordan) left derivation, zero (Jordan) product determined algebra, $C^{*}$-algebra, von Neumann algrbra.

* Corresponding author. 
$\delta_{1}=\frac{1}{2}(\delta+\hat{\delta})$ and $\delta_{2}=\frac{1}{2 i}(\delta-\hat{\delta})$. It is easy to show that $\delta_{1}$ and $\delta_{2}$ are both $*$-derivations. Similarly, we can define the $*$-Jordan derivations.

For $*$-derivations and $*$-Jordan derivations, in $[3,13,17,18]$, the authors characterize the following two conditions on a linear mapping $\delta$ from a $*$-algebra $\mathcal{A}$ into its $*$-bimodule $\mathcal{M}$ :

$$
\begin{aligned}
& \left(\mathbb{D}_{1}\right) a, b \in \mathcal{A}, a b^{*}=0 \Rightarrow a \delta(b)^{*}+\delta(a) b^{*}=0 ; \\
& \left(\mathbb{D}_{2}\right) a, b \in \mathcal{A}, a b^{*}=b^{*} a=0 \Rightarrow a \delta(b)^{*}+\delta(a) b^{*}=\delta(b)^{*} a+b^{*} \delta(a)=0 ;
\end{aligned}
$$

where $\mathcal{A}$ is a $C^{*}$-algebra, a zero product determined algebra or a group algebra $L^{1}(G)$.

Let $\mathcal{J}$ be an ideal of $\mathcal{A}$, we say that $\mathcal{J}$ is a right separating set or left separating set of $\mathcal{M}$ if for every $m$ in $\mathcal{M}, \mathcal{J} m=\{0\}$ implies $m=0$ or $m \mathcal{J}=\{0\}$ implies $m=$ 0 , respectively. We denote by $\mathfrak{J}(\mathcal{A})$ the subalgebra of $\mathcal{A}$ generated algebraically by all idempotents in $\mathcal{A}$.

In Section 2, we suppose that $\mathcal{A}$ is a $*$-algebra and $\mathcal{M}$ is a $*$ - $\mathcal{A}$-bimodule that satisfy one of the following conditions:

(1) $\mathcal{A}$ is a zero product determined Banach $*$-algebra with a bounded approximate identity and $\mathcal{M}$ is an essential Banach $*$ - $\mathcal{A}$-bimodule;

(2) $\mathcal{A}$ is a von Neumann algebra and $\mathcal{M}=\mathcal{A}$;

(3) $\mathcal{A}$ is a unital $*$-algebra and $\mathcal{M}$ is a unital $*$ - $\mathcal{A}$-bimodule with a left or right separating set $\mathcal{J} \subseteq \mathfrak{J}(\mathcal{A})$;

and we investigate whether the linear mappings from $\mathcal{A}$ into $\mathcal{M}$ satisfying the condition $\mathbb{D}_{1}$ characterize $*$-derivations. In particular, we generalize some results in $[13,17,18]$.

An $\mathcal{A}$-bimodule $\mathcal{M}$ is said to have the property $\mathbb{M}$, if there is an ideal $\mathcal{J} \subseteq \mathfrak{J}(\mathcal{A})$ of $\mathcal{A}$ such that

$$
\{m \in \mathcal{M}: x m x=0 \text { for every } x \in \mathcal{J}\}=\{0\} .
$$

It is clear that if $\mathcal{A}=\mathfrak{J}(\mathcal{A})$, then $\mathcal{M}$ has property $\mathbb{M}$.

For $*$-Jordan derivations, we can study the following conditions on a linear mapping $\delta$ from a $*$-algebra $\mathcal{A}$ into its $*$ - $\mathcal{A}$-bimodule $\mathcal{M}$ :

$$
\begin{aligned}
& \left(\mathbb{D}_{3}\right) a, b \in \mathcal{A}, a \circ b^{*}=0 \Rightarrow a \circ \delta(b)^{*}+\delta(a) \circ b^{*}=0 . \\
& \left(\mathbb{D}_{4}\right) a, b \in \mathcal{A}, a b^{*}=b^{*} a=0 \Rightarrow a \circ \delta(b)^{*}+\delta(a) \circ b^{*}=0 .
\end{aligned}
$$

It is obvious that the condition $\mathbb{D}_{2}$ or $\mathbb{D}_{3}$ implies the condition $\mathbb{D}_{4}$.

In Section 3, we suppose that $\mathcal{A}$ is a $*$-algebra and $\mathcal{M}$ is a $*$ - $\mathcal{A}$-bimodule that satisfy one of the following conditions:

(1) $\mathcal{A}$ is a unital zero Jordan product determined $*$-algebra and $\mathcal{M}$ is a unital *-A-bimodule;

(2) $\mathcal{A}$ is a unital $*$-algebra and $\mathcal{M}$ is a unital $*$ - $\mathcal{A}$-bimodule such that the property $\mathbb{M}$;

(3) $\mathcal{A}$ is a $C^{*}$-algebra (not necessary unital) and $\mathcal{M}$ is an essential Banach $*$ - $\mathcal{A}$ bimodule;

and we investigate whether the linear mappings from $\mathcal{A}$ into $\mathcal{M}$ satisfying the condition $\mathbb{D}_{3}$ or $\mathbb{D}_{4}$ characterize $*$-Jordan derivations. In particular, we improve some results in $[13,17,18]$. 


\section{2. *-DERIVATIONS ON SOME ALGEBRAS}

A (Banach) algebra $\mathcal{A}$ is said to be zero product determined if every (continuous) bilinear mapping $\phi$ from $\mathcal{A} \times \mathcal{A}$ into any (Banach) linear space $\mathcal{X}$ satisfying

$$
\phi(a, b)=0, \text { whenever } a b=0
$$

can be written as $\phi(a, b)=T(a b)$, for some (continuous) linear mapping $T$ from $\mathcal{A}$ into $\mathcal{X}$. In [7], M. Brešar shows that if $\mathcal{A}=\mathfrak{J}(\mathcal{A})$, then $\mathcal{A}$ is a zero product determined, and in [1], the authors prove that every $C^{*}$-algebra $\mathcal{A}$ is zero product determined.

Let $\mathcal{A}$ be a Banach $*$-algebra and $\mathcal{M}$ be a Banach $*$ - $\mathcal{A}$-bimodule. Denote by $\mathcal{M}^{\sharp \sharp}$ the second dual space of $\mathcal{M}$. In the following, we show that $\mathcal{M}^{\sharp \sharp}$ is also a Banach $*-\mathcal{A}$-bimodule.

Since $\mathcal{M}$ is a Banach $*-\mathcal{A}$-bimodule, $\mathcal{M}^{\sharp \sharp}$ turns into a dual Banach $\mathcal{A}$-bimodule with the operation defined by

$$
a \cdot m^{\sharp}=\lim _{\mu} a m_{\mu} \text { and } m^{\sharp \sharp} \cdot a=\lim _{\mu} m_{\mu} a
$$

for every $a$ in $\mathcal{A}$ and every $m^{\sharp \sharp}$ in $\mathcal{M}^{\sharp \sharp}$, where $\left(m_{\mu}\right)$ is a net in $\mathcal{M}$ with $\left\|m_{\mu}\right\| \leqslant$ $\left\|m^{\sharp \sharp}\right\|$ and $\left(m_{\mu}\right) \rightarrow m^{\sharp \sharp}$ in the weak ${ }^{*}$-topology $\sigma\left(\mathcal{M}^{\sharp \sharp}, \mathcal{M}^{\sharp}\right)$.

We define an involution $*$ in $\mathcal{M}^{\sharp \sharp}$ by

$$
\left(m^{\sharp \sharp}\right)^{*}(\rho)=\overline{m^{\sharp \sharp}\left(\rho^{*}\right)}, \rho^{*}(m)=\overline{\rho\left(m^{*}\right)},
$$

where $m^{\sharp \sharp}$ in $\mathcal{M}^{\sharp \sharp}, \rho$ in $\mathcal{M}^{\sharp}$ and $m$ in $\mathcal{M}$. Moreover, if $\left(m_{\mu}\right)$ is a net in $\mathcal{M}$ and $m^{\sharp \sharp}$ is an element in $\mathcal{M}^{\sharp \sharp}$ such that $m_{\mu} \rightarrow m^{\sharp \sharp}$ in $\sigma\left(\mathcal{M}^{\sharp \sharp}, \mathcal{M}^{\sharp}\right)$, then for every $\rho$ in $\mathcal{M}^{\sharp}$, we have that

$$
\rho\left(m_{\mu}\right)=m_{\mu}(\rho) \rightarrow m^{\sharp \sharp}(\rho) .
$$

It follows that

$$
\left(m_{\mu}^{*}\right)(\rho)=\rho\left(m_{\mu}^{*}\right)=\overline{\rho^{*}\left(m_{\mu}\right)} \rightarrow \overline{m^{\sharp \sharp}\left(\rho^{*}\right)}=\left(m^{\sharp \sharp}\right)^{*}(\rho)
$$

for every $\rho$ in $\mathcal{M}^{\sharp}$. It means that the involution $*$ in $\mathcal{M}^{\sharp \sharp}$ is continuous in $\sigma\left(\mathcal{M}^{\sharp \sharp}, \mathcal{M}^{\sharp}\right)$. Thus we can obtain that

$$
\left(a \cdot m^{\sharp \sharp}\right)^{*}=\left(\lim _{\mu} a m_{\mu}\right)^{*}=\lim _{\mu} m_{\mu}^{*} a^{*}=\left(m^{\sharp \sharp}\right)^{*} \cdot a^{*},
$$

Similarly, we can show that $\left(m^{\sharp \sharp} \cdot a\right)^{*}=a^{*} \cdot\left(m^{\sharp \sharp}\right)^{*}$. It implies that $\mathcal{M}^{\sharp \sharp}$ is a Banach $*$ - $\mathcal{A}$-bimodule.

Let $\mathcal{A}$ be a Banach $*$-algebra, a bounded approximate identity for $\mathcal{A}$ is a net $\left(e_{i}\right)_{i \in \Gamma}$ of self-adjoint elements in $\mathcal{A}$ such that $\lim _{i}\left\|a e_{i}-a\right\|=\lim _{i}\left\|e_{i} a-a\right\|=0$ for every $a$ in $\mathcal{A}$ and $\sup _{i \in \Gamma}\left\|e_{i}\right\| \leq k$ for some $k>0$.

In [18], H. Ghahramani and Z. Pan prove that if $\mathcal{A}$ is a unital zero product determined $*$-algebra and a linear mapping $\delta$ from $\mathcal{A}$ into itself satisfies the condition

$$
\left(\mathbb{D}_{1}\right) a, b \in \mathcal{A}, a b^{*}=0 \Rightarrow a \delta(b)^{*}+\delta(a) b^{*}=0
$$

then $\delta(a)=\Delta(a)+\delta(1) a$ for every $a$ in $\mathcal{A}$, where $\Delta$ is a $*$-derivation.

For general zero product determined Banach $*$-algebra with a bounded approximate identity, we have the following result. 
Theorem 2.1. Suppose that $\mathcal{A}$ is a zero product determined Banach *-algebra with a bounded approximate identity, and $\mathcal{M}$ is an essential Banach $*-\mathcal{A}$-bimodule. If $\delta$ is a continuous linear mapping from $\mathcal{A}$ into $\mathcal{M}$ such that

$$
a, b \in \mathcal{A}, a b^{*}=0 \Rightarrow a \delta(b)^{*}+\delta(a) b^{*}=0
$$

then there exist a $*$-derivation $\Delta$ from $\mathcal{A}$ into $\mathcal{M}^{\sharp \sharp}$ and an element $\xi$ in $\mathcal{M}^{\sharp \sharp}$ such that $\delta(a)=\Delta(a)+\xi \cdot$ a for every a in $\mathcal{A}$. Furthermore, $\xi$ can be chosen in $\mathcal{M}$ in each of the following cases:

(1) $\mathcal{A}$ is a unital $*$-algebra.

(2) $\mathcal{M}$ is a dual $*$-A-bimodule.

Proof. Let $\left(e_{i}\right)_{i \in \Gamma}$ be a bounded approximate identity of $\mathcal{A}$. Since $\delta$ is continuous, the net $\left(\delta\left(e_{i}\right)\right)_{i \in \Gamma}$ is bounded and we can assume that it converges to $\xi$ in $\mathcal{M}^{\sharp \sharp}$ with the topology $\sigma\left(\mathcal{M}^{\sharp \sharp}, \mathcal{M}^{\sharp}\right)$.

Since $\mathcal{M}$ is an essential Banach $*$ - $\mathcal{A}$-bimodule, we know that the nets $\left(e_{i} m\right)_{i \in \Gamma}$ and $\left(m e_{i}\right)_{i \in \Gamma}$ converge to $m$ with the norm topology for every $m$ in $\mathcal{M}$. Thus we have that

$$
\operatorname{Ann}_{\mathcal{M}}(\mathcal{A})=\{m \in \mathcal{M}: a m b=0 \text { for each } a, b \in \mathcal{A}\}=\{0\} .
$$

By the hypothesis, we can obtain that

$$
a, b, c \in \mathcal{A}, a b^{*}=b^{*} c=0 \Rightarrow a \delta(b)^{*} c=0 .
$$

It follows that

$$
a, b, c \in \mathcal{A}, a b=b c=0 \Rightarrow c^{*} b^{*}=b^{*} a^{*}=0 \Rightarrow c^{*} \delta(b)^{*} a^{*}=0 \Rightarrow a \delta(b) c=0 .
$$

By (2.1) and [1, Theorem 4.5], we know that

$$
\delta(a b)=\delta(a) b+a \delta(b)-a \cdot \xi \cdot b
$$

for each $a, b$ in $\mathcal{A}$, and $\xi$ can be chosen in $\mathcal{M}$ if $\mathcal{A}$ is a unital *-algebra or $\mathcal{M}$ is a dual $*$ - $\mathcal{A}$-bimodule.

Define a linear mapping $\Delta$ from $\mathcal{A}$ into $\mathcal{M}$ by

$$
\Delta(a)=\delta(a)-\xi \cdot a
$$

for every $a$ in $\mathcal{A}$. It is easy to show that $\Delta$ is a norm-continuous derivation from $\mathcal{A}$ into $\mathcal{M}^{\sharp \sharp}$ and we only need to show that $\Delta\left(b^{*}\right)=\Delta(b)^{*}$ for every $b$ in $\mathcal{A}$.

First we claim that $\Delta\left(e_{i}\right)=\delta\left(e_{i}\right)-\xi \cdot e_{i}$ converges to zero in $\mathcal{M}^{\sharp \sharp}$ with the topology $\sigma\left(\mathcal{M}^{\sharp \sharp}, \mathcal{M}^{\sharp}\right)$. In fact, since $\left(e_{i}\right)_{i \in \Gamma}$ is bounded in $\mathcal{A}$, we assume $\left(e_{i}\right)_{i \in \Gamma}$ converges to $\zeta$ in $\mathcal{A}^{\sharp \sharp}$ with the topology $\sigma\left(\mathcal{A}^{\sharp \sharp}, \mathcal{A}^{\sharp}\right)$. For every $m^{\sharp \sharp}$ in $\mathcal{M}^{\sharp \sharp}$, define

$$
m^{\sharp \sharp} \cdot \zeta=\lim _{i} m^{\sharp \sharp} \cdot e_{i} .
$$

Thus $m \cdot \zeta=m$ for every $m$ in $\mathcal{M}$. By [10, Proposition A.3.52], we know that the mapping $m^{\sharp \sharp} \mapsto m^{\sharp \sharp} \cdot \zeta$ from $\mathcal{M}^{\sharp \sharp}$ into itself is $\sigma\left(\mathcal{M}^{\sharp}, \mathcal{M}^{\sharp}\right)$-continuous, and by the $\sigma\left(\mathcal{M}^{\sharp \sharp}, \mathcal{M}^{\sharp}\right)$-denseness of $\mathcal{M}$ in $\mathcal{M}^{\sharp \sharp}$, we have that

$$
m^{\sharp \#} \cdot \zeta=m^{\sharp \sharp}
$$

for every $m^{\sharp \sharp}$ in $\mathcal{M}^{\sharp \sharp}$. Hence $\Delta\left(e_{i}\right)=\delta\left(e_{i}\right)-\xi \cdot e_{i}$ converges to zero in $\mathcal{M}^{\sharp \sharp}$ with the topology $\sigma\left(\mathcal{M}^{\sharp}, \mathcal{M}^{\sharp}\right)$. 
Next we prove $\Delta\left(b^{*}\right)=\Delta(b)^{*}$ for every $b$ in $\mathcal{A}$. By the definition of $\Delta$, we know that $a \Delta(b)^{*}+\Delta(a) b^{*}=0$ for each $a, b$ in $\mathcal{A}$ with $a b^{*}=0$. Define a bilinear mapping from $\mathcal{A} \times \mathcal{A}$ into $\mathcal{M}^{\sharp \sharp}$ by

$$
\phi(a, b)=a \Delta\left(b^{*}\right)^{*}+\Delta(a) b .
$$

Thus $a b=0$ implies $\phi(a, b)=0$. Since $\mathcal{A}$ is a zero product determined algebra, there exists a norm-continuous linear mapping $T$ from $\mathcal{A}$ into $\mathcal{M}^{\sharp \sharp}$ such that

$$
T(a b)=\phi(a, b)=a \Delta\left(b^{*}\right)^{*}+\Delta(a) b
$$

for each $a, b$ in $\mathcal{A}$. Let $b=e_{i}$ be in (2.3), we can obtain that

$$
T\left(a e_{i}\right)=a \Delta\left(e_{i}\right)^{*}+\Delta(a) e_{i} .
$$

By the continuity of $T$ and (2.2), it follows that $T(a)=\Delta(a)$ for every $a$ in $\mathcal{A}$. Thus

$$
T(a b)=\Delta(a b)=a \Delta\left(b^{*}\right)^{*}+\Delta(a) b .
$$

Since $\Delta$ is a derivation, we have that $a \Delta\left(b^{*}\right)^{*}=a \Delta(b)$ and $\Delta\left(b^{*}\right) a^{*}=\Delta(b)^{*} a^{*}$. Let $a=e_{i}$ and taking $\sigma\left(\mathcal{M}^{\sharp \sharp}, \mathcal{M}^{\sharp}\right)$-limits, by (2.2), it follows that $\Delta\left(b^{*}\right)=\Delta(b)^{*}$ for every $b$ in $\mathcal{A}$.

Let $G$ be a locally compact group. The group algebra and the measure convolution algebra of $G$, are denoted by $L^{1}(G)$ and $M(G)$, respectively. The convolution product is denote by - and the involution is denoted by $*$. It is well known that $M(G)$ is a unital Banach *-algebra, and $L^{1}(G)$ is a closed ideal in $M(G)$ with a bounded approximate identity. By [3, Lemma 1.1], we know that $L^{1}(G)$ is zero product determined. By [10, Theorem 3.3.15(ii)], it follows that $M(G)$ with respect to convolution product is the dual of $C_{0}(G)$ as a Banach $M(G)$-bimodule.

By [26, Corollary 1.2], we know that every continuous derivation $\Delta$ from $L^{1}(G)$ into $M(G)$ is an inner derivation, that is, there exists $\mu$ in $M(G)$ such that $\Delta(f)=f \cdot \mu-\mu \cdot f$ for every $f$ in $L^{1}(G)$. Thus by Theorem 2.1 , we can prove [17, Theorem 3.1(ii)] as follows.

Corollary 2.2. Let $G$ be a locally compact group. If $\delta$ is a continuous linear mapping from $L^{1}(G)$ into $M(G)$ such that

$$
f, g \in L^{1}(G), f \cdot g^{*}=0 \Rightarrow f \cdot \delta(g)^{*}+\delta(f) \cdot g^{*}=0
$$

then there are $\mu, \nu$ in $M(G)$ such that

$$
\delta(f)=f \cdot \mu-\nu \cdot f
$$

for every $f$ in $L^{1}(G)$ and $\operatorname{Re} \mu \in \mathcal{Z}(M(G))$.

Proof. By Theorem 2.1, we know that there exist a $*$-derivation $\Delta$ from $L^{1}(G)$ into $M(G)$ and an element $\xi$ in $M(G)$ such that $\delta(f)=\Delta(f)+\xi \cdot f$ for every $f$ in $L^{1}(G)$. By [26, Corollary 1.2], it follows that there exists $\mu$ in $M(G)$ such that $\Delta(f)=f \cdot \mu-\mu \cdot f$. Since $\Delta\left(f^{*}\right)=\Delta(f)^{*}$, we have that

$$
f^{*} \cdot \mu-\mu \cdot f^{*}=\mu^{*} \cdot f^{*}-f^{*} \cdot \mu^{*}
$$

for every $f$ in $L^{1}(G)$. By [3, Lemma 1.3(ii)], we know $\operatorname{Re} \mu=\frac{1}{2}\left(\mu+\mu^{*}\right) \in$ $\mathcal{Z}(M(G))$. Let $\nu=\mu-\xi$, from the definition of $\Delta$, we have that $\delta(f)=f \cdot \mu-\nu \cdot f$ for every $f$ in $L^{1}(G)$. 
For a general $C^{*}$-algebra $\mathcal{A}$, in [13], B. Fadaee and H. Ghahramani prove that if $\delta$ is a continuous linear mapping from $\mathcal{A}$ into its second dual space $\mathcal{A}^{\sharp \sharp}$ such that the condition $\mathbb{D}_{1}$, then there exist a $*$-derivation $\Delta$ from $\mathcal{A}$ into $\mathcal{A}^{\sharp \sharp}$ and an element $\xi$ in $\mathcal{A}^{\sharp \sharp}$ such that $\delta(a)=\Delta(a)+\xi a$ for every $a$ in $\mathcal{A}$.

In [1], the authors prove that every $C^{*}$-algebra $\mathcal{A}$ is zero product determined, and it is well known that $\mathcal{A}$ has a bounded approximate identity. Thus by Theorem 2.1, we can improve the result in [13] for any essential Banach $*$-bimodule.

Corollary 2.3. Suppose that $\mathcal{A}$ is a $C^{*}$-algebra and $\mathcal{M}$ is an essential Banach *-A-bimodule. If $\delta$ is a continuous linear mapping from $\mathcal{A}$ into $\mathcal{M}$ such that

$$
a, b \in \mathcal{A}, a b^{*}=0 \Rightarrow a \delta(b)^{*}+\delta(a) b^{*}=0
$$

then there exist a *-derivation $\Delta$ from $\mathcal{A}$ into $\mathcal{M}^{\sharp \sharp}$ and an element $\xi$ in $\mathcal{M}^{\sharp \sharp}$ such that $\delta(a)=\Delta(a)+\xi \cdot$ a for every a in $\mathcal{A}$. Furthermore, $\xi$ can be chosen in $\mathcal{M}$ in each of the following cases:

(1) $\mathcal{A}$ has an identity.

(2) $\mathcal{M}$ is a dual $*$-A-bimodule.

For von Neumann algebras, we have the following result.

Theorem 2.4. Suppose that $\mathcal{A}$ is a von Neumann algebra. If $\delta$ is a linear mapping from $\mathcal{A}$ into itself such that

$$
a, b \in \mathcal{A}, a b^{*}=0 \Rightarrow a \delta(b)^{*}+\delta(a) b^{*}=0,
$$

then $\delta(a)=\Delta(a)+\delta(1)$ a for every a in $\mathcal{A}$, where $\Delta$ is a $*$-derivation. In particular, $\delta$ is a*-derivation when $\delta(1)=0$.

Proof. Define a linear mapping $\Delta$ from $\mathcal{A}$ into $\mathcal{M}$ by

$$
\Delta(a)=\delta(a)-\delta(1) a
$$

for every $a$ in $\mathcal{A}$. In the following we show that $\Delta$ is a $*$-derivation. It is clear that $\Delta(1)=0$ and $a b^{*}=0$ can implies that $a \Delta(b)^{*}+\Delta(a) b^{*}=0$.

Case 1: Suppose that $\mathcal{A}$ is an abelian von Neumann algebra. First we show that $\Delta$ satisfies that

$$
a, b \in \mathcal{A}, a b=0 \Rightarrow a \Delta(b)=0 .
$$

It is well known that $\mathcal{A} \cong C(X)$, where $X$ is a compact Hausdorff space and $C(X)$ denotes the $C^{*}$-algebra of all continuous complex-valued functions on $X$. Thus we have that $a b=0$ if and only if $a b^{*}=0$ for each $a, b$ in $\mathcal{A}$. Indeed, let $f$ and $g$ be two functions in $C(X)$ corresponding to $a$ and $b$, respectively, we can obtain that

$$
a b^{*}=0 \Leftrightarrow f \cdot \bar{g}=0 \Leftrightarrow f \cdot g=0 \Leftrightarrow a b=0 .
$$

Let $a$ and $b$ be in $\mathcal{A}$ with $a b^{*}=a b=0$, we have that $a \Delta(b)^{*}+\Delta(a) b^{*}=0$. Multiply $a$ from the left side of above equation, we can obtain that $a^{2} \Delta(b)^{*}=0$. Let $f$ and $h$ be two functions in $C(X)$ corresponding to $a$ and $\Delta(b)$, then we have that

$$
0=f^{2} \bar{g}=f^{2} g=f g .
$$


It implies that $a \Delta(b)=0$. By [23, Theorem 3], we know that $\Delta$ is continuous. By [19, Lemma 2.5] and $\Delta(1)=0$, we know that $\Delta(a)=\Delta(1) a=0$ for every $a$ in $\mathcal{A}$.

Case 2: Suppose that $\mathcal{A} \cong M_{n}(\mathcal{B})$, where $\mathcal{B}$ is also a von Neumann algebra and $n \geqslant 2$. By $[6,7]$ we know that $\mathcal{A}$ is a zero product determined algebra. Thus by [18, Theorem 3.1] it follows that $\Delta$ is a $*$-derivation.

Case 3: Suppose that $\mathcal{A}$ is a general von Neumann algebra. It is well known that $\mathcal{A} \cong \sum_{i=1}^{n} \bigoplus \mathcal{A}_{i}$ ( $n$ is a finite integer or infinite), where each $\mathcal{A}_{i}$ coincides with either Case 1 or Case 2 . Denote the unit element of $\mathcal{A}_{i}$ by $1_{i}$ and the restriction of $\Delta$ in $\mathcal{A}_{i}$ by $\Delta_{i}$. Since $1_{i}\left(1-1_{i}\right)=0$ and $\Delta(1)=0$, we have that

$$
1_{i} \Delta\left(1-1_{i}\right)^{*}+\Delta\left(1_{i}\right)\left(1-1_{i}\right)=0 .
$$

It follows that

$$
-1_{i} \Delta\left(1_{i}\right)^{*}+\Delta\left(1_{i}\right)-\Delta\left(1_{i}\right) 1_{i}=0 .
$$

Multiplying $1_{i}$ from the left side of $(2.4)$ and by $1_{i} \Delta\left(1_{i}\right)=\Delta\left(1_{i}\right) 1_{i}$, we have that $1_{i} \Delta\left(1_{i}\right)^{*}=0$. It implies that $\Delta\left(1_{i}\right)=0$. For every $a$ in $\mathcal{A}$, we write $a=\sum_{i=1}^{n} a_{i}$ with $a_{i}$ in $\mathcal{A}_{i}$. Since $a_{i}\left(1-1_{i}\right)=0$, we have that $\Delta\left(a_{i}\right)\left(1-1_{i}\right)=0$, which means that $\Delta\left(a_{i}\right) \in \mathcal{A}_{i}$. Let $a_{i}, b_{i}$ be in $\mathcal{A}_{i}$ with $a_{i} b_{i}^{*}=0$, we have that

$$
\Delta\left(a_{i}\right) b_{i}^{*}+a_{i} \Delta\left(b_{i}\right)^{*}=\Delta_{i}\left(a_{i}\right) b_{i}^{*}+a_{i} \Delta_{i}\left(b_{i}\right)^{*}=0 .
$$

By Cases 1 and 2, we know that every $\Delta_{i}$ is a $*$-derivation. Thus $\Delta$ is a $*-$ derivation.

In the following, we characterize a linear mapping $\delta$ satisfies the condition $\mathbb{D}_{1}$ from a unital $*$-algebra into a unital $*$ - $\mathcal{A}$-bimodule with a right or left separating set $\mathcal{J} \subseteq \mathfrak{J}(\mathcal{A})$.

Lemma 2.5. [7, Theorem 4.1] Suppose that $\mathcal{A}$ is a unital algebra and $\mathcal{X}$ is a linear space. If $\phi$ is a bilinear mapping from $\mathcal{A} \times \mathcal{A}$ into $\mathcal{X}$ such that

$$
a, b \in \mathcal{A}, a b=0 \Rightarrow \phi(a, b)=0 \text {, }
$$

then we have that

$$
\phi(a, x)=\phi(a x, 1) \text { and } \phi(x, a)=\phi(1, x a)
$$

for every a in $\mathcal{A}$ and every $x$ in $\mathfrak{J}(\mathcal{A})$.

Theorem 2.6. Suppose that $\mathcal{A}$ is a unital *-algebra and $\mathcal{M}$ is a unital $*-\mathcal{A}$ bimodule with a right or left separating set $\mathcal{J} \subseteq \mathfrak{J}(\mathcal{A})$. If $\delta$ is a linear mapping from $\mathcal{A}$ into $\mathcal{M}$ such that

$$
a, b \in \mathcal{A}, a b^{*}=0 \Rightarrow a \delta(b)^{*}+\delta(a) b^{*}=0
$$

then $\delta(a)=\Delta(a)+\delta(1)$ a for every a in $\mathcal{A}$, where $\Delta$ is a $*$-derivation. In particular, $\delta$ is $a *$-derivation when $\delta(1)=0$.

Proof. Since $\mathcal{A}$ is a unital $*$-algebra and $\mathcal{M}$ is a unital $*$ - $\mathcal{A}$-bimodule, we know that $\mathcal{J} \subseteq \mathfrak{J}(\mathcal{A})$ is a right separating set of $\mathcal{M}$ if and only if $\mathcal{J}^{*}=\left\{x^{*}: x \in\right.$ $\mathcal{J}\} \subseteq \mathfrak{J}(\mathcal{A})$ is a left separating set of $\mathcal{M}$. Thus without loss of generality, we can assume that $\mathcal{J}$ is a left separating set of $\mathcal{A}$, otherwise, we replace $\mathcal{J}$ by $\mathcal{J}^{*}$. 
Define a linear mapping $\Delta$ from $\mathcal{A}$ into $\mathcal{M}$ by

$$
\Delta(a)=\delta(a)-\delta(1) a
$$

for every $a$ in $\mathcal{A}$. In the following we show that $\Delta$ is a $*$-derivation.

It is clear that $\Delta(1)=0$ and $a b^{*}=0$ can implies that $a \Delta(b)^{*}+\Delta(a) b^{*}=0$. Define a bilinear mapping $\phi$ from $\mathcal{A} \times \mathcal{A}$ into $\mathcal{M}$ by

$$
\phi(a, b)=a \Delta\left(b^{*}\right)^{*}+\Delta(a) b
$$

for each $a$ and $b$ in $\mathcal{A}$. By the assumption we know that $a b=0$ implies $\phi(a, b)=0$.

Let $a, b$ be in $\mathcal{A}$ and $x$ be in $\mathcal{J}$. By Lemma 2.5, we can obtain that

$$
\phi(x, 1)=\phi(1, x) \text { and } \phi(a, x)=\phi(a x, 1) .
$$

Hence we have the following two identities:

$$
x \Delta(1)^{*}+\Delta(x)=\Delta\left(x^{*}\right)^{*}+\Delta(1) x
$$

and

$$
a \Delta\left(x^{*}\right)^{*}+\Delta(a) x=a x \Delta(1)^{*}+\Delta(a x) .
$$

By (2.5) and $\Delta(1)=0$, we know that $\Delta(x)^{*}=\Delta\left(x^{*}\right)$. Thus by (2.6), it implies that

$$
\Delta(a x)=a \Delta(x)+\Delta(a) x .
$$

Similar to the proof of [4, Theorem 2.3], we can obtain that $\Delta(a b)=a \Delta(b)+\Delta(a) b$ for each $a$ and $b$ in $\mathcal{A}$.

It remains to show that $\Delta(a)^{*}=\Delta\left(a^{*}\right)$ for every $a$ in $\mathcal{A}$. Indeed, for every $a$ in $\mathcal{A}$ and every $x$ in $\mathcal{J}$, we have that $\Delta(a x)^{*}=\Delta\left((a x)^{*}\right)$. It implies that

$$
(\Delta(a) x+a \Delta(x))^{*}=\Delta\left(x^{*}\right) a^{*}+x^{*} \Delta\left(a^{*}\right) .
$$

Thus we can obtain that $x^{*}\left(\Delta(a)^{*}-\Delta\left(a^{*}\right)\right)=0$, hence $\left(\Delta(a)-\Delta\left(a^{*}\right)^{*}\right) x=0$. It follows that $\Delta(a)^{*}=\Delta\left(a^{*}\right)$ for every $a$ in $\mathcal{A}$.

Remark 1. Let $\mathcal{A}$ be a $*$-algebra, $\mathcal{M}$ be a $*$ - $\mathcal{A}$-bimodule, and $\delta$ is a linear mapping from $\mathcal{A}$ into $\mathcal{M}$. Similar to the condition $\mathbb{D}_{1}$ which we have characterized in Section 2:

$$
\left(\mathbb{D}_{1}\right) a, b \in \mathcal{A}, a b^{*}=0 \Rightarrow a \delta(b)^{*}+\delta(a) b^{*}=0,
$$

we can consider the condition $\mathbb{D}_{1}^{\prime}$

$$
\left(\mathbb{D}_{1}^{\prime}\right) a, b \in \mathcal{A}, a^{*} b=0 \Rightarrow a^{*} \delta(b)+\delta(a)^{*} b=0 .
$$

Through the minor modifications, we can obtain the corresponding results.

Remark 2. A linear mapping $\delta$ from $\mathcal{A}$ into $\mathcal{M}$ is called a local derivation if for every $a$ in $\mathcal{A}$, there exists a derivation $\delta_{a}$ (depending on $a$ ) from $\mathcal{A}$ into $\mathcal{M}$ such that $\delta(a)=\delta_{a}(a)$. It is clear that every local derivation satisfies the following condition:

$$
(\mathbb{H}) a, b, c \in \mathcal{A}, a b=b c=0 \Rightarrow a \delta(b) c=0 .
$$

In [1], the authors prove that every continuous linear mapping from a unital $C^{*}$ algebra into its unital Banach bimodule such that the condition $\mathbb{H}$ and $\delta(1)=0$ is a derivation. 
Let $\mathcal{A}$ be a $*$-algebra and $\mathcal{M}$ be a $*$ - $\mathcal{A}$-bimodule. The natural way to translate the condition $\mathbb{H}$ to the context of $*$-derivations is to consider the following condition

$$
\left(\mathbb{H}^{\prime}\right) a, b, c \in \mathcal{A}, a b^{*}=b^{*} c=0 \Rightarrow a \delta(b)^{*} c=0 .
$$

However, the conditions $\mathbb{H}^{\prime}$ and $\mathbb{H}$ are equivalent. Indeed, if condition $\mathbb{H}^{\prime}$ holds, we have that

$$
a, b, c \in \mathcal{A}, a b=b c=0 \Rightarrow c^{*} b^{*}=b^{*} a^{*}=0 \Rightarrow c^{*} \delta(b)^{*} a^{*}=0 \Rightarrow a \delta(b) c=0,
$$

and if the condition $\mathbb{H}$ holds, we have that

$$
a, b, c \in \mathcal{A}, a b^{*}=b^{*} c=0 \Rightarrow c^{*} b=b a^{*}=0 \Rightarrow c^{*} \delta(b) a^{*}=0 \Rightarrow a \delta(b)^{*} c=0 .
$$

It means that the condition $\mathbb{H}^{\prime}$ and $\delta(1)=0$ can not implies that $\delta$ is a *derivation.

\section{3. *-JORDAN DERIVATIONS ON SOME ALGEBRAS}

A (Banach) algebra $\mathcal{A}$ is said to be zero Jordan product determined if every (continuous) bilinear mapping $\phi$ from $\mathcal{A} \times \mathcal{A}$ into any (Banach) linear space $\mathcal{X}$ satisfying

$$
\phi(a, b)=0, \text { whenever } a \circ b=0
$$

can be written as $\phi(a, b)=T(a \circ b)$, for some (continuous) linear mapping $T$ from $\mathcal{A}$ into $\mathcal{X}$. In [5], we show that if $\mathcal{A}$ is a unital algebra with $\mathcal{A}=\mathfrak{J}(\mathcal{A})$, then $\mathcal{A}$ is a zero Jordan product determined algebra.

Theorem 3.1. Suppose that $\mathcal{A}$ is a unital zero Jordan product determined *algebra, and $\mathcal{M}$ is a unital $*$-A-bimodule. If $\delta$ is a linear mapping from $\mathcal{A}$ into $\mathcal{M}$ such that

$$
a, b \in \mathcal{A}, a \circ b^{*}=0 \Rightarrow a \circ \delta(b)^{*}+\delta(a) \circ b^{*}=0 \text { and } \delta(1) a=a \delta(1),
$$

then $\delta(a)=\Delta(a)+\delta(1)$ a for every $a$ in $\mathcal{A}$, where $\Delta$ is a $*$-Jordan derivation. In particular, $\delta$ is a $*$-Jordan derivation when $\delta(1)=0$.

Proof. Define a linear mapping $\Delta$ from $\mathcal{A}$ into $\mathcal{M}$ by $\Delta(a)=\delta(a)-\delta(1) a$ for every $a$ in $\mathcal{A}$. It is sufficient to show that $\Delta$ is a $*$-Jordan derivation.

It is clear that $\Delta(1)=0$, and by $\delta(1) a=a \delta(1)$ we have that

$$
a, b \in \mathcal{A}, a \circ b^{*}=0 \Rightarrow a \circ \Delta(b)^{*}+\Delta(a) \circ b^{*}=0 .
$$

Define a bilinear mapping from $\mathcal{A} \times \mathcal{A}$ into $\mathcal{M}$ by

$$
\phi(a, b)=a \circ \Delta\left(b^{*}\right)^{*}+\Delta(a) \circ b .
$$

Thus $a \circ b=0$ implies $\phi(a, b)=0$. Since $\mathcal{A}$ is a zero Jordan product determined algebra, we know that there exists a linear mapping $T$ from $\mathcal{A}$ into $\mathcal{M}$ such that

$$
T(a \circ b)=\phi(a, b)=a \circ \Delta\left(b^{*}\right)^{*}+\Delta(a) \circ b
$$

for each $a, b$ in $\mathcal{A}$. Let $a=1$ and $b=1$ be in (3.1), respectively. By $\Delta(1)=0$, we can obtain that

$$
T(a)=\Delta(a) \text { and } T(b)=\Delta\left(b^{*}\right)^{*} .
$$


It follows that $\Delta\left(a^{*}\right)=\Delta(a)^{*}$ for every $a$ in $\mathcal{A}$. By (3.1), we have that

$$
T(a \circ b)=\Delta(a \circ b)=\phi(a, b)=a \circ \Delta(b)+\Delta(a) \circ b .
$$

It means that $\Delta$ is a $*$-Jordan derivation.

In [5], we prove that the matrix algebra $M_{n}(\mathcal{B})(n \geq 2)$ is zero Jordan product determined, where $\mathcal{B}$ is a unital algebra. In [16], H. Ghahramani show that every Jordan derivation from $M_{n}(\mathcal{B})(n \geq 2)$ into its unital bimodule $\mathcal{M}$ is a derivation. Hence we have the following result.

Corollary 3.2. Suppose that $\mathcal{B}$ is a unital $*$-algebra, $M_{n}(\mathcal{B})$ is a matrix algebra with $n \geq 2$, and $\mathcal{M}$ is a unital $*-M_{n}(\mathcal{B})$-bimodule. If $\delta$ is a linear mapping from $M_{n}(\mathcal{B})$ into $\mathcal{M}$ such that

$$
a, b \in M_{n}(\mathcal{B}), a \circ b^{*}=0 \Rightarrow a \circ \delta(b)^{*}+\delta(a) \circ b^{*}=0 \text { and } \delta(1) a=a \delta(1),
$$

then $\delta(a)=\Delta(a)+\delta(1)$ a for every a in $M_{n}(\mathcal{B})$, where $\Delta$ is a *-derivation. In particular, $\delta$ is a*-derivation when $\delta(1)=0$.

Let $\mathcal{H}$ be a complex Hilbert space and $B(\mathcal{H})$ be the algebra of all bounded linear operators on $\mathcal{H}$. Suppose that $\mathcal{A}$ is a von Neumann algebra on $\mathcal{H}$ and $L S(\mathcal{A})$ the set of all locally measurable operators affiliated with the von Neumann algebra $\mathcal{A}$.

In [27], M. Muratov and V. Chilin prove that $L S(\mathcal{A})$ is a unital *-algebra and $\mathcal{A} \subset L S(\mathcal{A})$. By [25, Proposition 21.20, Exercise 21.18], we know that if $\mathcal{A}$ is a von Neumann algebra without direct summand of type $\mathrm{I}_{1}$, and $\mathcal{B}$ is a $*$-algebra with $\mathcal{A} \subseteq \mathcal{B} \subseteq L S(\mathcal{A})$, then $\mathcal{B} \cong \sum_{i=1}^{k} \bigoplus M_{n_{i}}\left(\mathcal{B}_{i}\right)$ ( $k$ is a finite integer or infinite), where $\mathcal{B}_{i}$ is a unital algebra. By Theorem 3.1, we have the following result.

Corollary 3.3. Suppose that $\mathcal{A}$ is a von Neumann algebra without direct summand of type $\mathrm{I}_{1}$, and $\mathcal{B}$ is a *-algebra with $\mathcal{A} \subseteq \mathcal{B} \subseteq L S(\mathcal{A})$. If $\delta$ is a linear mapping from $\mathcal{B}$ into $L S(\mathcal{A})$ such that

$$
a, b \in \mathcal{B}, a \circ b^{*}=0 \Rightarrow a \circ \delta(b)^{*}+\delta(a) \circ b^{*}=0 \text { and } \delta(1) a=a \delta(1),
$$

then $\delta(a)=\Delta(a)+\delta(1)$ a for every a in $\mathcal{B}$, where $\Delta$ is a $*$-Jordan derivation. In particular, $\delta$ is a $*$-Jordan derivation when $\delta(1)=0$.

For von Neumann algebras, by Corollary 3.2 and similar to the proof of Theorem 2.4, we can easily obtain the following result and we omit the proof.

Corollary 3.4. Suppose that $\mathcal{A}$ is a von Neumann algebra. If $\delta$ is a linear mapping from $\mathcal{A}$ into itself with such that

$$
a, b \in \mathcal{A}, a \circ b^{*}=0 \Rightarrow a \circ \delta(b)^{*}+\delta(a) \circ b^{*}=0 \text { and } \delta(1) a=a \delta(1),
$$

then $\delta(a)=\Delta(a)+\delta(1)$ a for every a in $\mathcal{A}$, where $\Delta$ is a $*$-derivation. In particular, $\delta$ is $a *$-derivation when $\delta(1)=0$.

Lemma 3.5. [5, Theorem 2.1] Suppose that $\mathcal{A}$ is a unital algebra and $\mathcal{X}$ is a linear space. If $\phi$ is a bilinear mapping from $\mathcal{A} \times \mathcal{A}$ into $\mathcal{X}$ such that

$$
a, b \in \mathcal{A}, a \circ b=0 \Rightarrow \phi(a, b)=0,
$$


then we have that

$$
\phi(a, x)=\frac{1}{2} \phi(a x, 1)+\frac{1}{2} \phi(x a, 1)
$$

for every a in $\mathcal{A}$ and every $x$ in $\mathfrak{J}(\mathcal{A})$.

Suppose that $\mathcal{A}$ is a unital algebra and $\mathcal{M}$ is a unital $\mathcal{A}$-bimodule satisfying that

$$
\{m \in \mathcal{M}: x m x=0 \text { for every } x \in \mathcal{J}\}=\{0\},
$$

where $\mathcal{J}$ is an ideal of $\mathcal{A}$ linear generated by idempotents in $\mathcal{A}$. In [15, Theorem 4.3], H. Ghahramani studies the linear mapping $\delta$ from $\mathcal{A}$ into $\mathcal{M}$ satisfies

$$
a, b \in \mathcal{A}, a \circ b=0 \Rightarrow a \circ \delta(b)+\delta(a) \circ b=0,
$$

and show that $\delta$ is a generalized Jordan derivation. In the following, we suppose that $\mathcal{J}$ is an ideal of $\mathcal{A}$ generated algebraically by all idempotents in $\mathcal{A}$, and have the following result.

Theorem 3.6. Suppose that $\mathcal{A}$ is a unital $*$-algebra, $\mathcal{M}$ is a unital $*$-A-bimodule, and $\mathcal{J} \subseteq \mathfrak{J}(\mathcal{A})$ is an ideal of $\mathcal{A}$ such that

$$
\{m \in \mathcal{M}: x m x=0 \text { for every } x \in \mathcal{J}\}=\{0\} .
$$

If $\delta$ is a linear mapping from $\mathcal{A}$ into $\mathcal{M}$ such that

$$
a, b \in \mathcal{A}, a \circ b^{*}=0 \Rightarrow a \circ \delta(b)^{*}+\delta(a) \circ b^{*}=0 \text { and } \delta(1) a=a \delta(1),
$$

then $\delta(a)=\Delta(a)+\delta(1)$ a for every a in $\mathcal{A}$, where $\Delta$ is a $*$-Jordan derivation. In particular, $\delta$ is a $*$-Jordan derivation when $\delta(1)=0$.

Proof. Let $\widehat{\mathcal{J}}$ be an algebra generated algebraically by $\mathcal{J}$ and $\mathcal{J}^{*}$. Since $\mathcal{J} \subseteq \mathfrak{J}(\mathcal{A})$ is an ideal of $\mathcal{A}$, it is easy to show that $\widehat{\mathcal{J}} \subseteq \mathfrak{J}(\mathcal{A})$ is also an ideal of $\mathcal{A}$, and such that

$$
\{m \in \mathcal{M}: x m x=0 \text { for every } x \in \widehat{\mathcal{J}}\}=\{0\} .
$$

Thus without loss of generality, we can assume that $\mathcal{J}$ is a self-adjoint ideal of $\mathcal{A}$, otherwise, we may replace $\mathcal{J}$ by $\widehat{\mathcal{J}}$.

Define a linear mapping $\Delta$ from $\mathcal{A}$ into $\mathcal{M}$ by

$$
\Delta(a)=\delta(a)-\delta(1) a
$$

for every $a$ in $\mathcal{A}$. In the following we show that $\Delta$ is a $*$-derivation.

It is clear that $\Delta(1)=0$, and by $\delta(1) a=a \delta(1)$ we have that $a \circ b^{*}=0$ implies that $a \circ \Delta(b)^{*}+\Delta(a) \circ b^{*}=0$.

Define a bilinear mapping $\phi$ from $\mathcal{A} \times \mathcal{A}$ into $\mathcal{M}$ by

$$
\phi(a, b)=a \circ \Delta\left(b^{*}\right)^{*}+\Delta(a) \circ b
$$

for each $a$ and $b$ in $\mathcal{A}$. By the assumption we know that $a \circ b=0$ implies $\phi(a, b)=0$.

Let $a, b$ be in $\mathcal{A}$ and $x$ be in $\mathcal{J}$. By Lemma 3.5, we can obtain that

$$
\phi(x, 1)=\phi(1, x) .
$$

It follows that

$$
x \circ \Delta(1)^{*}+\Delta(x) \circ 1=1 \circ \Delta\left(x^{*}\right)^{*}+\Delta(1) \circ x .
$$


By (3.2) and $\Delta(1)=0$, we know that $\Delta(x)^{*}=\Delta\left(x^{*}\right)$. Again by Lemma 3.5, it follows that

$$
a \circ \Delta\left(x^{*}\right)^{*}+\Delta(a) \circ x=\frac{1}{2}[\Delta(a x) \circ 1+\Delta(x a) \circ 1] .
$$

By (3.3) and $\Delta(x)^{*}=\Delta\left(x^{*}\right)$, it is easy to show that

$$
\Delta(a \circ x)=a \circ \Delta(x)+\Delta(a) \circ x .
$$

Next, we prove that $\Delta$ is a Jordan derivation.

Define $\{a, m, b\}=a m b+b m a$ and $\{a, b, m\}=\{m, b, a\}=a b m+m b a$ for each $a, b$ in $\mathcal{A}$ and every $m$ in $\mathcal{M}$. Let $a$ be in $\mathcal{A}$ and $x, y$ be in $\mathcal{M}$.

By the technique of the proof of [15, Theorem 4.3] and (3.4), we have the following two identities:

$$
\Delta\{x, a, y\}=\{\Delta(x), a, y\}+\{x, \Delta(a), y\}+\{x, a, \Delta(y)\},
$$

and

$$
\Delta\left\{x, a^{2}, y\right\}=\left\{\Delta(x), a^{2}, y\right\}+\{x, a \circ \Delta(a), y\}+\left\{x, a^{2}, \Delta(y)\right\} .
$$

On the other hand, by (3.5) we have that

$$
\Delta\left\{x, a^{2}, x\right\}=\left\{\Delta(x), a^{2}, x\right\}+\left\{x, \Delta\left(a^{2}\right), x\right\}+\left\{x, a^{2}, \Delta(x)\right\} .
$$

By comparing (3.6) and (3.7), it follows that $\left\{x, \Delta\left(a^{2}\right), x\right\}=\{x, a \circ \Delta(a), x\}$. That is $x\left(\Delta\left(a^{2}\right)-a \circ \Delta(a)\right) x=0$. By the assumption, it implies that $\Delta\left(a^{2}\right)-a \circ \Delta(a)=0$ for every $a$ in $\mathcal{A}$.

It remains to show that $\Delta(a)^{*}=\Delta\left(a^{*}\right)$ for every $a$ in $\mathcal{A}$. Indeed, for every $a$ in $\mathcal{A}$ and every $x$ in $\mathcal{J}$, we have that $\Delta(x a x)^{*}=\Delta\left((x a x)^{*}\right)$. Since $\Delta$ is a Jordan derivation, it implies that

$$
(\Delta(x) a x+x \Delta(a) x+x a \Delta(x))^{*}=\Delta\left(x^{*}\right) a^{*} x^{*}+x^{*} \Delta\left(a^{*}\right) x^{*}+x^{*} a^{*} \Delta\left(x^{*}\right) .
$$

Thus we can obtain that $x^{*}\left(\Delta(a)^{*}-\Delta\left(a^{*}\right)\right) x^{*}=0$. Since $\mathcal{J}$ is a self-adjoint ideal of $\mathcal{A}$, it follows that $\Delta(a)^{*}=\Delta\left(a^{*}\right)$.

Let $\mathcal{A}$ be a $C^{*}$-algebra and $\mathcal{M}$ be a Banach $*$ - $\mathcal{A}$-bimodule. Denote by $\mathcal{A}^{\sharp \sharp}$ and $\mathcal{M}^{\sharp \sharp}$ the second dual space of $\mathcal{A}$ and $\mathcal{M}$, respectively. By [11, p.26], we can define a product $\diamond$ in $\mathcal{A}^{\sharp \sharp}$ by

$$
a^{\sharp \sharp} \diamond b^{\sharp \sharp}=\lim _{\lambda} \lim _{\mu} \alpha_{\lambda} \beta_{\mu}
$$

for each $a^{\sharp \sharp}, b^{\sharp \sharp}$ in $\mathcal{A}^{\sharp \sharp}$, where $\left(\alpha_{\lambda}\right)$ and $\left(\beta_{\mu}\right)$ are two nets in $\mathcal{A}$ with $\left\|\alpha_{\lambda}\right\| \leqslant\left\|a^{\sharp \sharp}\right\|$ and $\left\|\beta_{\mu}\right\| \leqslant\left\|b^{\sharp \sharp}\right\|$, such that $\alpha_{\lambda} \rightarrow a^{\sharp \sharp}$ and $\beta_{\mu} \rightarrow b^{\sharp \sharp}$ in the weak*-topology $\sigma\left(\mathcal{A}^{\sharp \sharp}, \mathcal{A}^{\sharp}\right)$. Moreover, we can define an involution $*$ in $\mathcal{A}^{\sharp \sharp}$ by

$$
\left(a^{\sharp \sharp}\right)^{*}(\rho)=\overline{a^{\sharp \sharp}\left(\rho^{*}\right)}, \rho^{*}(a)=\overline{\rho\left(a^{*}\right)},
$$

where $a^{\sharp \sharp}$ in $\mathcal{A}^{\sharp \sharp}, \rho$ in $A^{\sharp}$ and $a$ in $\mathcal{A}$. By [22, p.726], we know that $\mathcal{A}^{\sharp \sharp}$ is a von Neumann algebra under the product $\diamond$ and the involution $*$.

Since $\mathcal{M}$ is a Banach $\mathcal{A}$-bimodule, $\mathcal{M}^{\sharp \sharp}$ turns into a dual Banach $\left(\mathcal{A}^{\sharp \sharp}, \diamond\right)$ bimodule with the operation defined by

$$
a^{\sharp \sharp} \cdot m^{\sharp \sharp}=\lim _{\lambda} \lim _{\mu} a_{\lambda} m_{\mu} \text { and } m^{\sharp \sharp} \cdot a^{\sharp}=\lim _{\mu} \lim _{\lambda} m_{\mu} a_{\lambda}
$$


for every $a^{\sharp \sharp}$ in $\mathcal{A}^{\sharp \sharp}$ and every $m^{\sharp \sharp}$ in $\mathcal{M}^{\sharp \sharp}$, where $\left(a_{\lambda}\right)$ is a net in $\mathcal{A}$ with $\left\|a_{\lambda}\right\| \leqslant$ $\left\|a^{\sharp \sharp}\right\|$ and $\left(a_{\lambda}\right) \rightarrow a^{\sharp \sharp}$ in $\sigma\left(\mathcal{A}^{\sharp \sharp}, \mathcal{A}^{\sharp}\right),\left(m_{\mu}\right)$ is a net in $\mathcal{M}$ with $\left\|m_{\mu}\right\| \leqslant\left\|m^{\sharp \sharp}\right\|$ and $\left(m_{\mu}\right) \rightarrow m^{\sharp \sharp}$ in $\sigma\left(\mathcal{M}^{\sharp \sharp}, \mathcal{M}^{\sharp}\right)$.

We remarked, in the discussion preceding Theorem 2.1, that $\mathcal{M}^{\sharp \sharp}$ has an involution $*$ and it is continuous in $\sigma\left(\mathcal{M}^{\sharp}, \mathcal{M}^{\sharp}\right)$. By [1, p.553], we know that every continuous bilinear map $\varphi$ from $\mathcal{A} \times \mathcal{M}$ into $\mathcal{M}$ is Arens regular, which means that

$$
\lim _{\lambda} \lim _{\mu} \varphi\left(a_{\lambda}, m_{\mu}\right)=\lim _{\mu} \lim _{\lambda} \varphi\left(a_{\lambda}, m_{\mu}\right)
$$

for every $\sigma\left(\mathcal{A}^{\sharp \sharp}, \mathcal{A}^{\sharp}\right)$-convergent net $\left(a_{\lambda}\right)$ in $\mathcal{A}$ and every $\sigma\left(\mathcal{M}^{\sharp \sharp}, \mathcal{M}^{\sharp}\right)$-convergent net $\left(m_{\mu}\right)$ in $\mathcal{M}$. Thus we can obtain that

$$
\left(a^{\sharp \sharp} \cdot m^{\sharp \sharp}\right)^{*}=\left(\lim _{\lambda} \lim _{\mu} a_{\lambda} m_{\mu}\right)^{*}=\lim _{\lambda} \lim _{\mu} m_{\mu}^{*} a_{\lambda}^{*}=\lim _{\mu} \lim _{\lambda} m_{\mu}^{*} a_{\lambda}^{*}=\left(m^{\sharp \sharp}\right)^{*} \cdot\left(a^{\sharp \sharp}\right)^{*},
$$

where $\left(a_{\lambda}\right)$ is a net in $\mathcal{A}$ with $\left(a_{\lambda}\right) \rightarrow a^{\sharp \sharp}$ in $\sigma\left(\mathcal{A}^{\sharp \sharp}, \mathcal{A}^{\sharp}\right)$ and $\left(m_{\mu}\right)$ is a net in $\mathcal{M}$ with $\left(m_{\mu}\right) \rightarrow m^{\sharp \sharp}$ in $\sigma\left(\mathcal{M}^{\sharp \sharp}, \mathcal{M}^{\sharp}\right)$. Similarly, we can show that $\left(m^{\sharp \sharp} \cdot a^{\sharp \sharp}\right)^{*}=$ $\left(a^{\sharp \sharp}\right)^{*} \cdot\left(m^{\sharp \sharp}\right)^{*}$. It implies that $\mathcal{M}^{\sharp \sharp}$ is a Banach $*-\mathcal{A}^{\sharp \#-b i m o d u l e . ~}$

A projection $p$ in $\mathcal{A}^{\sharp \sharp}$ is called open if there exists an increasing net $\left(a_{\alpha}\right)$ of positive elements in $\mathcal{A}$ such that $p=\lim _{\alpha} a_{\alpha}$ in the weak*-topology of $\mathcal{A}^{\sharp \sharp}$. If $p$ is open, we say the projection $1-p$ is closed.

For a unital $C^{*}$-algebra, we have the following result.

Theorem 3.7. Suppose that $\mathcal{A}$ is a unital $C^{*}$-algebra and $\mathcal{M}$ is a unital Banach *-A-bimodule. If $\delta$ is a continuous linear mapping from $\mathcal{A}$ into $\mathcal{M}$ such that $\delta(1) a=a \delta(1)$ for every $a$ in $\mathcal{A}$, then the following three statements are equivalent:

(1) $a, b \in \mathcal{A}, a \circ b^{*}=0 \Rightarrow a \circ \delta(b)^{*}+\delta(a) \circ b^{*}=0$;

(2) $a, b \in \mathcal{A}, a b^{*}=b^{*} a=0 \Rightarrow a \circ \delta(b)^{*}+\delta(a) \circ b^{*}=0$;

(3) $\delta(a)=\Delta(a)+\delta(1)$ a for every a in $\mathcal{A}$, where $\Delta$ is a $*$-derivation from $\mathcal{A}$ into $\mathcal{M}$.

Proof. It is clear that $(1) \Rightarrow(2)$ and $(3) \Rightarrow(1)$. It is sufficient the prove that $(2) \Rightarrow(3)$.

Define a linear mapping $\Delta$ from $\mathcal{A}$ into $\mathcal{M}$ by $\Delta(a)=\delta(a)-\delta(1) a$ for every $a$ in $\mathcal{A}$. It is sufficient to show that $\Delta$ is a $*$-derivation. First we prove that $\Delta\left(a^{*}\right)=\Delta(a)^{*}$ for every $a$ in $\mathcal{A}$.

By assumption, we can easily to show that

$$
a, b \in \mathcal{A}, a b^{*}=b^{*} a=0 \Rightarrow a \circ \Delta(b)^{*}+\Delta(a) \circ b^{*}=0 \text { and } \Delta(1)=0,
$$

In the following, we verify $\Delta(b)=\Delta(b)^{*}$ for every self-adjoint element $b$ in $\mathcal{A}$.

Since $\Delta$ is a norm continuous linear mapping form $\mathcal{A}$ into $\mathcal{M}$, we know that $\Delta^{\sharp \sharp}:\left(\mathcal{A}^{\sharp \sharp}, \diamond\right) \rightarrow \mathcal{M}^{\sharp \sharp}$ is the weak ${ }^{*}$-continuous extension of $\Delta$ to the double duals of $\mathcal{A}$ and $\mathcal{M}$.

Let $b$ be a non-zero self-adjoint element in $\mathcal{A}, \sigma(b) \subseteq[-\|b\|,\|b\|]$ be the spectrum of $b$ and $r(b) \in \mathcal{A}^{\sharp \sharp}$ be the range projection of $b$.

Denote by $\mathcal{A}_{b}$ the $C^{*}$-subalgebra of $\mathcal{A}$ generated by $b$, and by $C(\sigma(b))$ the $C^{*}$ algebra of all continuous complex-valued functions on $\sigma(b)$. By Gelfand theory we know that there is an isometric * isomorphism between $\mathcal{A}_{b}$ and $C(\sigma(b))$. 
For every $n$ in $\mathbb{N}$, let $p_{n}$ be the projection in $\mathcal{A}_{b}^{\sharp \sharp} \subseteq \mathcal{A}^{\sharp \sharp}$ corresponding to the characteristic function $\chi_{\left(\left[-\|b\|,-\frac{1}{n}\right] \cup\left[\frac{1}{n},\|b\|\right]\right) \cap \sigma(b)}$ in $C(\sigma(b))$, and let $b_{n}$ be in $\mathcal{A}_{b}$ such that

$$
b_{n} p_{n}=p_{n} b_{n}=b_{n}=b_{n}^{*} \text { and }\left\|b_{n}-b\right\|<\frac{1}{n} .
$$

By [28, Section 1.8], we know that $\left(p_{n}\right)$ converges to $r(b)$ in the strong*-topology of $\mathcal{A}^{\sharp \sharp}$, and hence in the weak*-topology.

It is well known that $p_{n}$ is a closed projection in $\mathcal{A}_{b}^{\sharp \sharp} \subseteq \mathcal{A}^{\sharp \sharp}$ and $1-p_{n}$ is an open projection in $\mathcal{A}_{b}^{\sharp \sharp}$. Thus there exists an increasing net $\left(z_{\lambda}\right)$ of positive elements in $\left(\left(1-p_{n}\right) \mathcal{A}^{\sharp \sharp}\left(1-p_{n}\right)\right) \cap \mathcal{A}$ such that

$$
0 \leq z_{\lambda} \leq 1-p_{n}
$$

and $\left(z_{\lambda}\right)$ converges to $1-p_{n}$ in the weak*-topology of $\mathcal{A}^{\sharp \sharp}$. Since

$$
0 \leq\left(\left(1-p_{n}\right)-z_{\lambda}\right)^{2} \leq\left(1-p_{n}\right)-z_{\lambda} \leq\left(1-p_{n}\right),
$$

we have that $\left(z_{\lambda}\right)$ also converges to $1-p_{n}$ in the strong*-topology of $\mathcal{A}^{\sharp \sharp}$.

By $b_{n}=b_{n}^{*}$ and $z_{\lambda} b_{n}=b_{n} z_{\lambda}=0$, it follows that

$$
z_{\lambda} \circ \Delta^{\sharp \sharp}\left(b_{n}\right)^{*}+\Delta^{\sharp \sharp}\left(z_{\lambda}\right) \circ b_{n}=0 .
$$

Taking weak*-limits in (3.8) and since $\Delta^{\sharp \#}$ is weak*-continuous, we have that

$$
\left(1-p_{n}\right) \circ \Delta^{\sharp \sharp}\left(b_{n}\right)^{*}+\Delta^{\sharp \sharp}\left(\left(1-p_{n}\right)\right) \circ b_{n}=0 .
$$

Since $\left(p_{n}\right)$ converges to $r(b)$ in the weak*-topology of $\mathcal{A}^{\sharp \sharp}$ and $\left(b_{n}\right)$ converges to $b$ in the norm-topology of $\mathcal{A}$, by (3.9), we have that

$$
(1-r(b)) \circ \Delta^{\sharp \sharp}(b)^{*}+\Delta^{\sharp \sharp}(1-r(b)) \circ b=0 .
$$

Since the range projection of every power $b^{m}$ with $m \in \mathbb{N}$ coincides with the $r(b)$, and by (3.10), it follows that

$$
(1-r(b)) \circ \Delta^{\sharp \sharp}\left(b^{m}\right)^{*}+\Delta^{\sharp \sharp}(1-r(b)) \circ b^{m}=0
$$

for every $m \in \mathbb{N}$, and by the linearity and norm continuity of the product we have that

$$
(1-r(b)) \circ \Delta^{\sharp \sharp}(z)^{*}+\Delta^{\sharp \sharp}(1-r(b)) \circ z=0
$$

for every $z=z^{*}$ in $\mathcal{A}_{b}$. A standard argument involving weak*-continuity of $\Delta^{\sharp}$ gives

$$
(1-r(b)) \circ \Delta^{\sharp \sharp}(r(b))^{*}+\Delta^{\sharp \sharp}(1-r(b)) \circ r(b)=0 .
$$

By (3.11), we can obtain that

$$
\left(\Delta^{\sharp \sharp}(r(b))^{*}+\Delta^{\sharp \sharp}(r(b))-\Delta^{\sharp \sharp}(1)\right) \circ r(b)=2 \Delta^{\sharp \sharp}(r(b))^{*} .
$$

By $\Delta(1)=0$, we have that $\Delta^{\sharp \sharp}(1)=0$. It implies that

$$
\Delta^{\sharp \sharp}(r(b))^{*}=\Delta^{\sharp \sharp}(r(b)) \text {. }
$$

It is clear that every characteristic function

$$
p=\chi_{([-\|b\|,-\alpha] \cup[\alpha,\|b\|]) \cap \sigma(b)}
$$


in $C_{0}(\sigma(b))^{\sharp \sharp}$ with $0<\alpha<\|b\|$, is the range projection of a function in $C(\sigma(b))$. Moreover, every projection of the form

$$
q=\chi_{([-\beta,-\alpha] \cup[\alpha, \beta]) \cap \sigma(b)}
$$

in $C_{0}(\sigma(b))^{\sharp \sharp}$ with $0<\alpha<\beta<\|b\|$ can be written as the difference of two projections of the type in (3.13).

Since $\mathcal{A}_{b}$ and $C(\sigma(b))$ are isometric $*$ isomorphism, and by $\Delta^{\sharp}(r(b))^{*}=\Delta^{\sharp \sharp}(r(b))$ for range projection of $b$ in $\mathcal{A}^{\sharp \sharp}$, we have that $\Delta^{\sharp \sharp}(p)^{*}=\Delta^{\sharp \sharp}(p)$ for every projection $p$ of the type in (3.13). It follows that $\Delta^{\sharp \sharp}(q)^{*}=\Delta^{\sharp \sharp}(q)$ for every projection $q$ of the type in (3.14).

It is well known that $b$ can be approximated in norm by finite linear combinations of mutually orthogonal projections $q_{j}$ of the type in (3.14), and $\Delta$ is continuous, we have that $\Delta(b)^{*}=\Delta(b)$. Thus for every $a$ in $\mathcal{A}$, we can obtain that $\Delta(a)^{*}=\Delta(a)$.

By the assumption, it follows that

$$
a, b \in \mathcal{A}, a b=b a=0 \Rightarrow a \circ \Delta(b)+\Delta(a) \circ b=0 .
$$

By [2, Theorem 4.1], we know that $\Delta$ is a $*$-derivation.

In the following we consider general $C^{*}$-algebras $\mathcal{A}$. Let $\left(e_{i}\right)_{i \in \Gamma}$ be a bounded approximate identity of $\mathcal{A}, \mathcal{M}$ be an essential Banach $*$ - $\mathcal{A}$-bimodule, and $\delta$ be a continuous linear mapping from $\mathcal{A}$ into $\mathcal{M}$, then $\left(\delta\left(e_{i}\right)\right)_{i \in \Gamma}$ is bounded and we can assume that it converges to $\xi$ in $\mathcal{M}^{\sharp \sharp}$ with the topology $\sigma\left(\mathcal{M}^{\sharp}, \mathcal{M}^{\sharp}\right)$. It follows the next result.

Theorem 3.8. Suppose that $\mathcal{A}$ is a $C^{*}$-algebra (not necessary unital) and $\mathcal{M}$ is an essential Banach $*$-A-bimodule. If $\delta$ is a continuous linear mapping from $\mathcal{A}$ into $\mathcal{M}$ such that $\xi \cdot a=a \cdot \xi$ for every $a$ in $\mathcal{A}$, then the following three statements are equivalent:

(1) $a, b \in \mathcal{A}, a \circ b^{*}=0 \Rightarrow a \circ \delta(b)^{*}+\delta(a) \circ b^{*}=0$;

(2) $a, b \in \mathcal{A}, a b^{*}=b^{*} a=0 \Rightarrow a \circ \delta(b)^{*}+\delta(a) \circ b^{*}=0$

(3) $\delta(a)=\Delta(a)+\xi \cdot$ a for every a in $\mathcal{A}$, where $\Delta$ is a *-derivation from $\mathcal{A}$ into $\mathcal{M}^{\sharp \#}$.

Proof. It is clear that $(1) \Rightarrow(2)$ and $(3) \Rightarrow(1)$. It is only need to prove that $(2) \Rightarrow(3)$.

Define a linear mapping $\Delta$ from $\mathcal{A}$ into $\mathcal{M}^{\sharp \sharp}$ by

$$
\Delta(a)=\delta(a)-\xi \cdot a
$$

for every $a$ in $\mathcal{A}$. It is sufficient to show that $\Delta$ is a $*$-derivation. that

By the definition of $\Delta$ and $\xi \cdot a=a \cdot \xi$ for every $a$ in $\mathcal{A}$, we can easily to show

$$
a, b \in \mathcal{A}, a b^{*}=b^{*} a=0 \Rightarrow a \circ \Delta(b)^{*}+\Delta(a) \circ b^{*}=0 .
$$

By [10, Proposition 2.9.16], we know that $\left(e_{i}\right)_{i \in \Gamma}$ converges to the identity 1 in $\mathcal{A}^{\sharp \sharp}$ with the topology $\sigma\left(\mathcal{A}^{\sharp \sharp}, \mathcal{A}^{\sharp}\right)$. By the proof of Theorem 2.1, we know that 
$\Delta\left(e_{i}\right)=\delta\left(e_{i}\right)-e_{i} \cdot \xi$ converges to zero in $\mathcal{M}^{\sharp \sharp}$ with the topology $\sigma\left(\mathcal{M}^{\sharp \sharp}, \mathcal{M}^{\sharp}\right)$, and we can obtain that

$$
m^{\sharp \#} \cdot 1=m^{\sharp \sharp}
$$

for every $m^{\sharp \sharp}$ in $\mathcal{M}^{\sharp \sharp}$. Since $\mathcal{M}^{\sharp \sharp}$ is a Banach $*$ - $\mathcal{A}^{\sharp \sharp}$-bimodule, we have that

$$
1 \cdot m^{\sharp}=m^{\sharp \#}
$$

for every $m^{\sharp \sharp}$ in $\mathcal{M}^{\sharp \sharp}$. Since $\Delta$ is a norm-continuous linear mapping form $\mathcal{A}$ into $\mathcal{M}^{\sharp \sharp}, \Delta^{\sharp \sharp}:\left(\mathcal{A}^{\sharp \sharp}, \diamond\right) \rightarrow \mathcal{M}^{\sharp \sharp \sharp \sharp}$ is the weak* ${ }^{*}$-continuous extension of $\Delta$ to the double duals of $\mathcal{A}$ and $\mathcal{M}^{\sharp \sharp}$ such that $\Delta^{\sharp \sharp}(1)=0$.

By [10, Proposition A.3.52], we know that the mapping $m^{\sharp \sharp \sharp} \mapsto m^{\sharp \sharp \sharp} \cdot 1$ from $\mathcal{M}^{\sharp \sharp \sharp}$ into itself is $\sigma\left(\mathcal{M}^{\sharp \sharp \sharp}, \mathcal{M}^{\sharp \sharp \sharp}\right)$-continuous, and by the $\sigma\left(\mathcal{M}^{\sharp \sharp \sharp}, \mathcal{M}^{\sharp \sharp \sharp}\right)$ denseness of $\mathcal{M}^{\sharp \sharp}$ in $\mathcal{M}^{\sharp \# \#}$, we have that

$$
m^{\sharp \# \#} \cdot 1=m^{\sharp \# \#}
$$

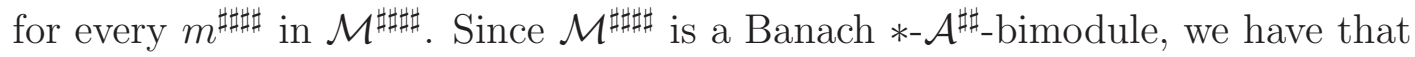

$$
1 \cdot m^{\sharp \# \#}=m^{\sharp \# \# \#}
$$

for every $m^{\sharp \# \#}$ in $\mathcal{M}^{\sharp \sharp \sharp \sharp}$.

Finally, we use the same proof of Theorem 3.7 and show that $\Delta$ is a $*$-derivation from $\mathcal{A}$ into $\mathcal{M}^{\sharp \sharp}$.

Remark 3. In [12], A. Essaleh and A. Peralta introduce the concept of a triple derivation on $C^{*}$-algebras. Suppose that $\mathcal{A}$ is a $C^{*}$-algebra. Let $a, b$ and $c$ be in $\mathcal{A}$, define the ternary product by $\{a, b, c\}=\frac{1}{2}\left(a b^{*} c+c b^{*} a\right)$. A linear mapping $\delta$ from $\mathcal{A}$ into itself is called a triple derivation if

$$
\delta\{a, b, c\}=\{\delta(a), b, c\}+\{a, \delta(b), c\}+\{a, b, \delta(c)\}
$$

for each $a, b$ and $c$ in $\mathcal{A}$. Let $z$ be an element in $\mathcal{A}$. $\delta$ is called triple derivation at $z$ if

$$
a, b, c \in \mathcal{A},\{a, b, c\}=z \Rightarrow \delta(z)=\{\delta(a), b, c\}+\{a, \delta(b), c\}+\{a, b, \delta(c)\} .
$$

In [12], A. Essaleh and A. Peralta prove that every continuous linear mapping $\delta$ which is triple derivations at zero from a unital $C^{*}$-algebra into itself with $\delta(1)=0$ is a $*$-derivation.

On the other hand, it is apparent to show that if $\delta$ is triple derivation at zero, then $\delta$ satisfies that

$$
a, b \in \mathcal{A}, a b^{*}=b^{*} a=0 \Rightarrow a \circ \delta(b)^{*}+\delta(a) \circ b^{*}=0 .
$$

Thus Theorem 3.7 generalizes [12, Corollary 2.10].

Remark 4. In [8], M. Brešar and J. Vukman introduce the left derivations and Jordan left derivations. A linear mapping $\delta$ from an algebra $\mathcal{A}$ into its bimodule $\mathcal{M}$ is called a left derivation if $\delta(a b)=a \delta(b)+b \delta(a)$ for each $a, b$ in $\mathcal{A}$; and $\delta$ is called a Jordan left derivation if $\delta(a \circ b)=2 a \delta(b)+2 b \delta(a)$ for each $a, b$ in $\mathcal{A}$.

Let $\mathcal{A}$ be a $*$-algebra and $\mathcal{M}$ be a $*$ - $\mathcal{A}$-bimodule. A left derivation (Jordan left derivation) $\delta$ from $\mathcal{A}$ into $\mathcal{M}$ is called a $*$-left derivation (*-Jordan left derivation) if $\delta\left(a^{*}\right)=\delta(a)^{*}$ for every $a$ in $\mathcal{A}$. 
We also can investigate the following conditions on a linear mapping $\delta$ from $\mathcal{A}$ into $\mathcal{M}$ :

$$
\begin{aligned}
& \left(\mathbb{J}_{1}\right) a, b \in \mathcal{A}, a b^{*}=0 \Rightarrow a \delta(b)^{*}+b^{*} \delta(a)=0 \\
& \left(\mathbb{J}_{2}\right) a, b \in \mathcal{A}, a \circ b^{*}=0 \Rightarrow a \delta(b)^{*}+b^{*} \delta(a)=0 \\
& \left(\mathbb{J}_{3}\right) a, b \in \mathcal{A}, a b^{*}=b^{*} a=0 \Rightarrow a \delta(b)^{*}+b^{*} \delta(a)=0 .
\end{aligned}
$$

Acknowledgement. This research was partly supported by the National Natural Science Foundation of China (Grant Nos. 11801342, 11801005, 11871021); Natural Science Foundation of Shaanxi Province (Grant No. 2020JQ-693); Scientific research plan projects of Shannxi Education Department (Grant No. 19JK0130).

\section{REFERENCES}

[1] J. Alaminos, M. Brešar, J. Extremera and A. Villena. Maps preserving zero products, Studia Math., 193 (2009) 131-159.

[2] J. Alaminos, M. Brešar, J. Extremera and A. Villena. Characterizing Jordan maps on $C^{*}{ }_{-}$ algebras through zero products, Proc. Edinburgh Math. Soc., 53 (2010), 543-555.

[3] J. Alaminos, M. Brešar, J. Extremera and A. Villena. Orthogonality preserving linear maps on group algebras, Math. Proc. Camb. Phil. Soc., 158 (2015), 493-504.

[4] G. An and J. Li. Characterizations of linear mappings through zero products or zero Jordan products, Electron. J. Linear Algebra, 31 (2016), 408-424.

[5] G. An, J. Li and J. He. Zero Jordan product determined algebras, Linear Algebra Appl., 475 (2015), 90-93.

[6] M. Brešar. Characterizing homomorphisms, derivations and multipliers in rings with idempotents, Proc. Roy. Soc. Edinburgh Sect. A, 137 (2007), 9-21.

[7] M. Brešar. Multiplication algebra and maps determined by zero products, Linear Multilinear Algebra, 60 (2012), 763-768.

[8] M. Brešar and J. Vukman. On left derivations and related mappings, Proc. Amer. Math. Soc., 110 (1990), 7-16.

[9] J. Cuntz. On the continuity of Semi-Norms on operator algebras, Math. Ann., 220 (1976), 171-183.

[10] H. Dales. Banach Algebras and Automatic Continuity, London Math. Soc. Monog. Ser. 24, Oxford Univ. Press, New York, 2000.

[11] H. Dales, F. Ghahramani and N. Grønbæk. Derivations into iterated duals of Banach algebra, Studia Math., 128 (1998), 19-54.

[12] A. Essaleh and A. Peralta. Linear maps on $C^{*}$-algebras which are derivations or triple derivations at a point, Linear Algebra Appl., 538 (2018), 1-21.

[13] B. Fadaee and H. Ghahramani. Linear maps behaving like derivations or anti-derivations at orthogonal elements on $C^{*}$-algebras, arXiv: $1907.03594 \mathrm{v} 1$.

[14] P. Fillmore and D. Topping. Operator algebras generated by projections, Duke Math. J., 34 (1967), 333-336.

[15] H. Ghahramani. On derivations and Jordan derivations through zero products, Oper. Matrices, 8 (2014), 759-771.

[16] H. Ghahramani. Characterizing Jordan derivations of matrix rings through zero products, Math. Slovaca, 65 (2015), 1277-1290.

[17] H. Ghahramani. Linear maps on group algebras determined by the action of the derivations or anti-derivations on a set of orthogonal element, Results Math., 73 (2018), no. 4, Art. 133, 14pp.

[18] H. Ghahramani and Z. Pan. Linear maps on *-algebras acting on orthogonal element like derivations or anti-derivations, Filomat, 13 (2018), 4543-4554.

[19] J. He, J. Li and W. Qian. Characterizations of centrelizers and derivations on some algebras, J. Korean Math. Soc., 54 (2017), 685-696. 
[20] S. Hejazian and A. Niknam. Modules, annihilators and module derivations of J $B^{*}$-algebras, Indian J. Pure Appl. Math., 27 (1996), 129-140.

[21] B. Johnson. Symmetric amenability and the nonexistence of Lie and Jordan derivations Math. Proc. Cambd. Philos. Soc., 120 (1996), 455-473.

[22] R. Kadison and J. Ringrose. Fundamentals of the Theory of Operator Algebras, I, Pure Appl. Math. 100, Academic Press, New York, 1983.

[23] R. Kantrowitz and M. Neumann. Disjointness preserving and local operators on algebras of differentiable functions, Glasgow Math. J., 43 (2001), 295-309.

[24] A. Kishimoto. Dissipations and Derivations, Commun. Math. Phys., 47 (1976), 25-32.

[25] T. Lam. A first course in noncommutative rings, Springer-Verlag, New York, 1991.

[26] V. Losert. The derivation problem for group algebras Ann. Math., 168 (2008), 221-246.

[27] M. Muratov and V. Chilin. Algebras of measurable and locally measurable operators, Kyiv, Pratse In-ty matematiki NAN ukraini., 69 (2007), 390 pp, (Russian).

[28] S. Sakai. $C^{*}$-Algebras and $W^{*}$-Algebras, Springer-Verlag, Berlin, 1971.

${ }^{1}$ Department of Mathematics, Shannxi University of Science and Technology, XI'AN 710021, CHINA.

E-mail address: anguangyu310@163.com

2 Department of Mathematics, Anhui Polytechnic University, Wuhu 241000, CHINA.

E-mail address: hejun_12@163.com

3* Department of Mathematics, East China University of Science and TechNology, Shanghai 200237, China.

E-mail address: jiankuili@yahoo.com 